Copyright (@ 2009 Institute of Electrical and electronics Engineers, Inc.

All Rights reserved.

Personal use of this material, including one hard copy reproduction, is permitted.

Permission to reprint, republish and/or distribute this material in whole or in part for any other purposes must be obtained from the IEEE.

For information on obtaining permission, send an e-mail message to stds-igr@ieee.org.

By choosing to view this document, you agree to all provisions of the copyright laws protecting it.

Individual documents posted on this site may carry slightly different copyright restrictions.

For specific document information, check the copyright notice at the beginning of each document. 


\title{
VEHICLE-TRACK MODELLING FOR RAIL CORRUGATION INITIATION INVESTIGATION
}

\author{
Yan Quan Sun \\ Scott Simson \\ Centre for Railway Engineering \\ Central Queensland University \\ North Rockhampton, QLD 4702 \\ Australia
}

Tel: 617 49309287, Email mailto:y.q.sun@acqu.edu.au

\begin{abstract}
As a part of Australian Rail CRC Project $\# 82$ - Bogie Rotation Friction Management, the investigation on rail corrugation initiation has been carried out. This paper reports the progress made on this research. At present, the literature review and the vehicle-track modelling for rail corrugation have been finished. A three-dimensional vehicle-track system dynamics model is developed for the simulations, in which the vehicle dynamics is described using up to 78 degrees of freedom (DOF). The wheelsets are considered as flexible bodies. The track is modeled as one-layered, two rail beams on elastic foundation or two-layered, sleepers included structure. The effect of wheel-rail profiles, wheel-rail interface conditions, curved track parameters, wheelset design parameters and centre bowl rotation friction on the rail corrugations will be examined.
\end{abstract}

\section{INTRODUCTION}

The rail corrugations have been observed for a century and at present the variety of theories advanced to explain their occurrence match the variety of the phenomenon itself. Generally, the phenomenon is the more or less periodic irregularities of the running surfaces on rails, which are often visible to the naked eye, and cause the high dynamic loads between wheels and rails, deteriorating the track and vehicle components and resulting in the high noise.

It is known that the corrugation is a phenomenon of great practical concems to the railway industry. Substantial savings could be made if corrugations of rails and wheels could be prevented rather than simply treated. For the past 30 years, many theoretical and experimental studies have been conducted by railway researchers. There are several hypotheses put forward to explain the corrugation formation based the theoretical and experimental analyses. Grassie and Kalousek (1993) [1] considered that the wheel-rail dynamic load P2 resonance; sleeper resonance; flexural resonance; torsional resonance of wheelset mainly contributed the six types of rail corrugations respectively, namely, "heavy haul" and "light rail" corrugations, corrugations on resiliently "booted sleepers", "contact fatigue" corrugations in curves, "rutting" and "roaring rails". It was assumed that some initial track geometry irregularities and/or wheel and rail defects in combination with other factors such as traction, creep and the friction characteristics at the wheel-rail contact excited dynamic loads which caused damage of some types, thereby modifying the initial profiles of rails or wheels and forming corrugations.

Valdivia (1987) [2] explained the initiating mechanism of corrugations with the assumption that high frequency vibrations of wheelset-track system could be excited by the stochastical roughness of the railhead surface and then these vibrations in return influenced the shape of the railhead surface such that only waves within a narrow band of tength are filtered out. Hempelmann et al. (1992) . [3] considered that the different dynamic behaviour of track at midspan or above a sleeper at the first vertical pinned-pinned mode with a frequency of $1080 \mathrm{~Hz}$ was one of the main reasons for the formation of rail corrugation. Knothe and Ripke (1989) [4] applied the same track modelling as that by Hempelmann et al. (1992) to analyse the effect of some parameters on the growth rate of rail corrugation and pointed out that the maximum corrugation growth rate was not only depending on the frequency but also on the vehicle speed. If a speed of $180 \mathrm{~km} / \mathrm{h}(50 \mathrm{~m} / \mathrm{s})$ is taken, the first maximum occurs for a comugation wavelength of $4 \sim 6$

$\mathrm{cm}\left(L=\frac{V}{f}=\frac{50}{1080}=4.63 \mathrm{~cm}\right)[4]$.

Tessilly and Vincent (1991) [5] reported their investigations conceming rail cornugations on the Paris Mass Transportation Authority network. Two types of corrugations were observed: (1). Wavelength of $8 \sim 12 \mathrm{~cm}$; concrete track; express trains (frequency range of $200-300 \mathrm{~Hz}$ at $90 \mathrm{~km} / \mathrm{h}$ ), 
and (2). Wavelength of more than $12 \mathrm{~cm}$; on ballasted track (typical frequency range $60 \sim 100 \mathrm{~Hz}$ at $40 \mathrm{~km} / \mathrm{h}$ ). In both cases coincidence between some natural vibrating modes of the wheelset and the dynamic behaviour of track could explain corrugation growth.

Kalousek and Johnson (1992) [6] thought that the simplest hypothesis to explain the short-wavelength corrugation, namely, that corrugation arose from the vertical dynamic behaviour of the wheel-rail system excited by the initially aperiodic railhead roughness was found to be inadequate. The reasonable hypothesis would be that short-wavelength corrugation arose from a combination of the dynamic behaviour of the track normally and in its plane, with a initially aperiodic railhead roughness giving rise to variations in the normal load and consequently also to variations in the magnitude of slip and wear in the contact area. A common feature of such hypothesis had been that corrugation was exacerbated by high quasi-static creepage at the wheel-rail contact, as might arise during curving or more generally from misaligned wheelsets. It was specially pointed out that corrugation happened not only on the running surface of rails but also on the tread surface of wheels. Similar conclusion was also made by Grassie (1996) [7] that for the short-wavelength corrugation, excitation of the vertical dynamic behaviour of vehicle and track by railhead roughness is an insufficient explanation for the initiation of this type of corrugation.

In the early 1980s, Clark and Foster (1983) [8] investigated the self-excited vibration characteristics of a flexible wheelset and track system under high creepage conditions. Meanwhile, the non-linear vibrations excited by track variations and the stick-slip vibrations excited by wheel-rail creep forces were also considered. It was suggested that these mechanisms might exist separately or in combination to provide possible explanation for the formation of long- and short-wavelength corrugations on the running surface of railway rails.

Ahlback and Daniels (1991) [9] pointed out that the basic differences in long- and short-wavelength corrugation generation processes would require somewhat different approaches in control. In both types a vertical wheel-track dynamic resonance with resulting high contact stresses was a necessary ingredient. In the case ' of long-wavelength corrugations on heavy haul railways, even the static wheel loads approached the strength limits of the rail. The added dynamic loads could then induce plastic deformation and regenerate surface profile damage. The more complex shortwavelength corrugations required combinations of wheel and track parameters that induced unstable stick-slip oscillations with possible heat and metallurgical damage at the rail surface.

Suda and Iguchi (1989) [10] pointed out that corrugations would be caused by contact resonance, some self-excited vibrations with slip between wheel and rail and some vibrations of wheelset excited by track irregularities. However, the corrugation growth depended on the system (wheelset-track) coupled natural frequency. The rigid support of rail gave the long-wavelength corrugation in connection with the first coupled natural frequency while the soft support introduced the short-wavelength wavelength depending on the second coupled natural frequency. Hays and Tucker (1991) [11] reported their investigation of the interaction of the torsional dynamics of the wheelset and the longitudinal dynamics of the track, coupled through the contact patch, as a possible source of rail corrugation wear. For their investigation on the corrugation of low rail in a curved track, Matsumoto et al. (2002) [12] concluded that the corrugation was caused by:

a. Excessively large creepage between wheel and rail, which was caused by insufficiency of rolling radius difference between high rail and low rail side wheels or large attack angle between wheel and rail,

b. Oscillation of wheel load (vertical contact force) which was caused by vertical vibration including Hertzian contact stiffness or rail rolling vibration,

c. Stick-slip between wheel and rail accompanying with the wheel load oscillation.

For the mechanism of short-wavelength corrugation, $\mathrm{Wu}$ and Thompson (2002) [13] predicted the corrugation growth at different frequencies or wavelengths for typical train speeds through combining the features of multiple wheel-rail interactions, unsteady rolling contact and the detailed wear calculations.

Based on the above reviewed literature, the corrugation formation on rails and wheels could be classified as following four types:

- System resonances:

Here system refers to vehicle-track system. Although some wheelset-track or bogie-track systems are able to predict resonances for some corrugations, the interactions between wheelsets or bogies are neglected and the natural frequencies of system might be inaccurately determined. Special track geometry irregularities and/or rail defects, generating large oscillation of vertical wheel-rail contact force can be induced with a natural frequency of system, especially when there is less damping in the track structure. Generally for the vehicles with heavy wheel load, the static wheel load approaches the strength limits of the rail. The added dynamic contact forces with large amplitude could then induce plastic deformation on the running surface of rails.

- Self-excitation of wheel-rail stick-slip process:

From non-linear vibration theory, the conditions of selfexcitation generation for a system should be: (1). A system with external energy supplier, (2). A system able to store and release energy, and (3). A system with a switch for storing and releasing energy. Corresponding to a vehicletrack system, especiaily a wheel-rail subsystem, the first condition is that the energy of wheelsets and rails is provided by locomotives. For the second condition, the axles of wheelsets have an ability to store and release torsional and bending energy. For the third condition, the switch is the positive and negative friction characteristics between wheels and rails - stick and slip.

Explanation can be given in Fig. 1 that shows the wheelrail stick-slip cycle. In the positive friction zone, wheel 
sticks on rail from A to B at the large creepage situation, and axle stores torsional and bending energy. B is a saturation point from which wheel starts to slip on rail. Because wheel starts slipping, creepage suddenly increases and jumps to $C$. In the negative friction zone, wheel slips on rail and friction force decreases. After axle releases energy, creepage decreases to D and wheel stops slipping. Due to the ending of wheel's slipping, creepage suddenly jumps to $A$ and wheel sticks on rail again.

In our assumption, if torsional energy of axles is mainly stored and released, corrugation might be longitudinally formatted; and if bending energy of axles is stored and released, wheel squeal might be induced laterally.

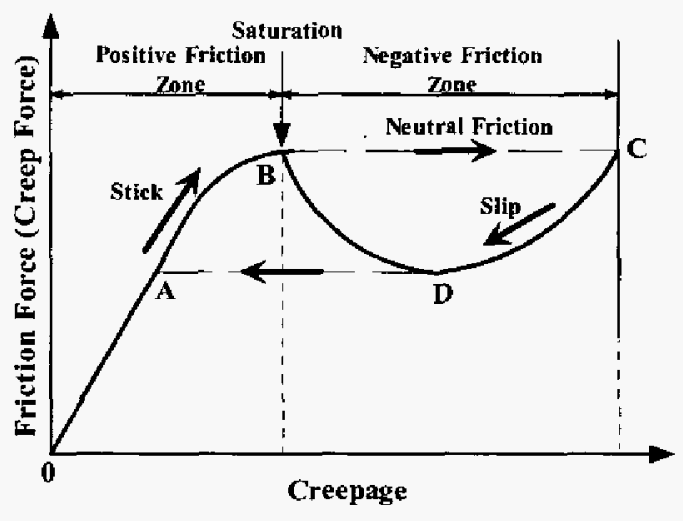

Figure 1. Wheel-rall Stick-slip Cycle [8]

- Combination of self-excitation of wheel-rail stick-slip and system resonance: system resonance may come from local component resonance such as some wheelset modes and track modes (e.g. sleeper resonance).

- Wheel-rail contact fatigue: based on the work done by Grassie and Kalousek (1993) [1].

In order to examine the effects of wheel-rail profiles, wheel-rail interface conditions, curved track parameters, wheelset design parameters and centre bowl rotation friction on the rail corrugation initiation, a three-dimensional wagon-track system dynamics model for rail corrugation was developed. In this model, wagon movements were described using 78 degrees of freedom (DOF). Specially, the wheelset was considered to be flexible and 9 DOF were used to illustrate movements of wheelset including torsion and bending about longitudinal and vertical directions. Track modelling was considered as onelayer (two rail beams on elastic foundation) or two-layer structure (sleeper beams were included). For wheel-rail interface, Hertz contact theory was used to describe the normal contact of wheel-rail and Kalker's creep theory to describe longitudinal, lateral and spin creeps between wheel and rail. Detailed description for this model will be given in this paper, and some simulations related to rail corrugation will be also given in this paper.

\section{VEHICLE-TRACK MODELLING}

DYNAMICS

SYSTEM

In this section, modelling of vehicle-track dynamics system for the investigation on corrugation initiation is given. The dynamic vehicle-track system is divided into three subsystems - vehicle, track and wheel-rail contact, which are described in the following subsections respectively.

\subsection{Vehicle Subsystem}

The model is for a three-piece bogie vehicle as generally seen for freight or coal vehicles. The vehicle subsystem includes one car body which is described as rigid body using six DOF (lateral, vertical and longitudinal displacements, and roll, pitch and yaw rotations), two bolsters each of them is described as rigid body using six DOF, four sideframes each of them is deseribed as rigid body using six DOF, four wheelsets each of them is described as rigid and flexural body using nine DOF (lateral and vertical and longitudinal displacement of whole wheelset, and two wheel masses in roll, pitch and yaw rotations coupled through some stiffness coefficients corresponding to the first natural torsional and bending modes of wheelset). The secondary and primary suspensions are modelled as non-linear spring and damper elements. Figure 2. (a), (b), (c) and (d) show these components modelling respectively.

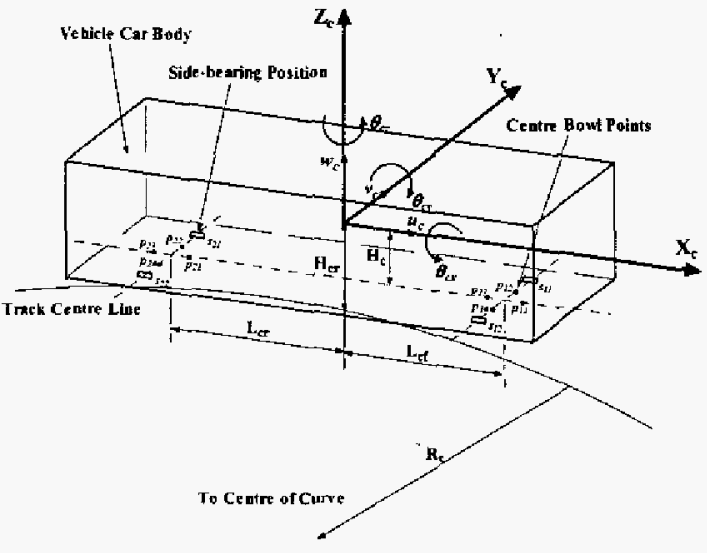

(a) Car Body

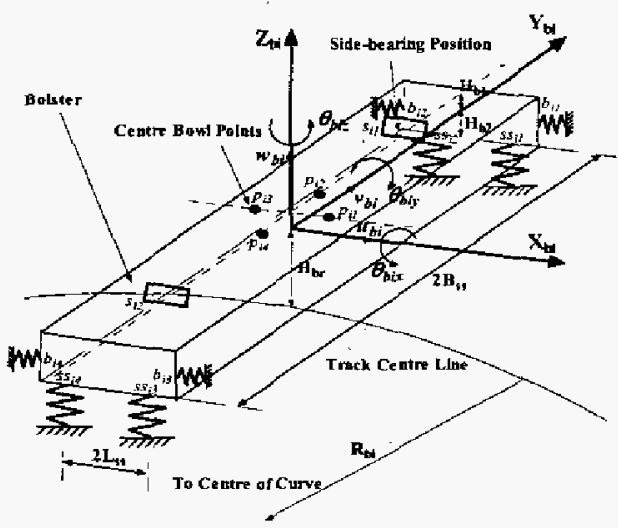

(b) Bolster 


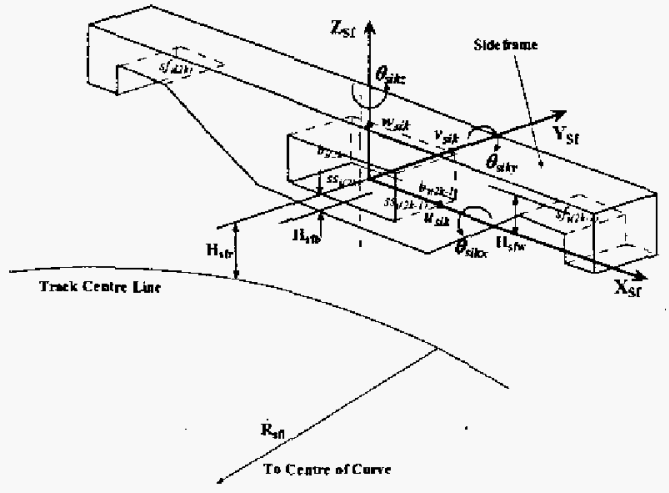

(c) Sideframe

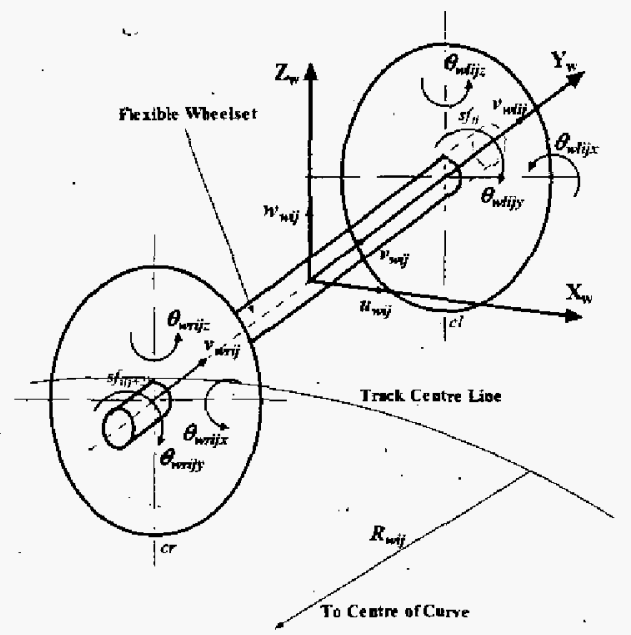

(d) Wheelset

Figure 2. Vehicle Modelling

It is expected that the corrugations can be thoroughly investigated using such vehicle subsystem modelling. Meanwhile, the effects of centre bow friction (bogie yaw) (for the centre bowl connections, 4-point contacts are used for the modelling) on the initiation of corrugations can be examined. Before the vehicle subsystem modelling is made, the natural frequencies and vibrating modes of a typical wheelset should be calculated using some professional finite element analysis packages such as ABAQUS.

\subsection{Track Subsystem}

The track subsystem is considered as the discretely supported distributed-parameter track with two layers as shown in Figure. 3 (a) and (b). The track components are assembled exactly as the conventional ballasted track structure used in most of the heavy haul railway network. The track subsystem comprises of two rails, $n_{s}$ sleepers, $4 \times n_{s}$ fastener and pad assembly, ballast arranged in two layers.

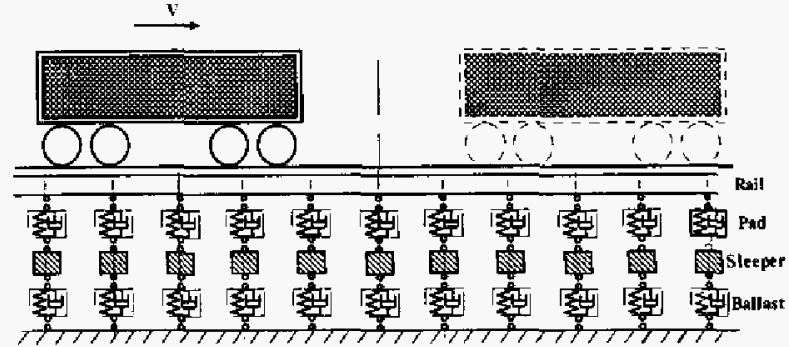

(a) Longitudinal View

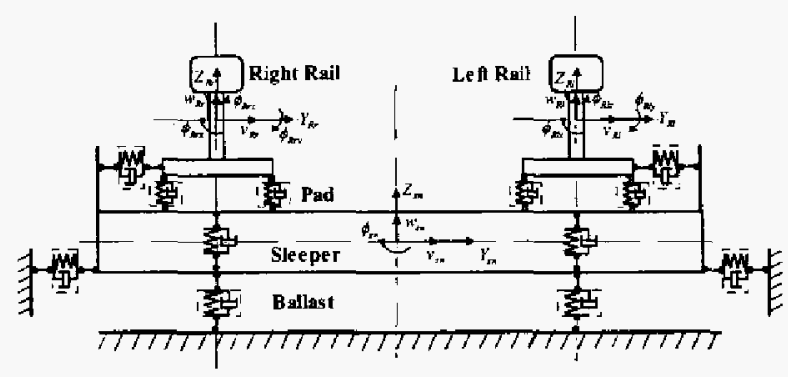

(b) Lateral View

Figure 3. Track Modelling

Such track modelling can be further simplified. The layer for sleepers can be ignored. Modified track modelling will be two rail beams on elastic foundation.

\subsection{Wheel-Rail Interface Subsystem}

In this subsystem, the creep forces and normal forces in the wheel-rail interface are determined according to Kalker creep theory and Hertz contact theory respectively.

The creep forces $\left(F_{c}\right)$ are determined according to the Fig. 1 , which shows the relationship between the creep force and the creepage $(\xi)$. The longitudinal creepage, the lateral creepage and the spin creepage are expressed as:

$$
\left\{\begin{array}{l}
\xi_{k x}=\left[\dot{R}_{w k} \cdot e_{1 k}-V\left(r_{k} / r_{0}\right) \cos \theta_{u z}\right] / V \\
\xi_{k v}=\left[\left(\dot{R}_{w k}-\dot{R}_{R k}\right) \cdot e_{2 k}\right] / V \\
\xi_{k s p}=\left[\left(\omega_{w k}-\omega_{R k}\right) \cdot e_{3 k}\right] / V
\end{array}\right.
$$

in which the subscript $k=l$ or $r$ representing left and right wheels, $\left(e_{1 k}, e_{2 k}, e_{3 k}\right)$ is the wheel-rail contact point axle unit vectors, $R_{w k}$ is the position vectors of the left or the right contact points in the wheelset equilibrium coordinate system, $R_{R k}$ is the position vectors of the left or the right contact points in the rail equilibrium coordinate system, $r_{k}$ is the wheel radius at the contact points, $\omega_{w k}$ and $\omega_{R k}$ are the angular velocities of wheel and rail respectively. 
For very small creepages, Kalker's linear creep theory is used to develop relationships between the creep forces and the creepages. These are given as follows:

Longitudinal creep force: $F_{c x}=-f_{33} \xi_{x}$,

Lateral creep force: $F_{c y}=-f_{11} \xi_{y}-f_{12} \xi_{s p}$,

Spin creep moment: $M_{c z}=f_{12} \xi_{y}-f_{22} \xi_{s p}$.

in which $f_{11}, f_{12}, f_{22}$, and $f_{33}$ are the creep coefficients. In Fig. 1, the $0 \mathrm{AB}$ section before the saturation at $\mathrm{B}$ shows the relationship between creepage and creep force, which is determined by Kalkar's creep theory and revised by JohnsonVermeuten's approach for creep without spin, and described as follows:

The resultant force $F_{r e}$ as follows:

$$
F_{r e}=\left(F_{c x}^{2}+F_{c y}^{2}\right)^{1 / 2}
$$

The limiting resultant force $\bar{F}_{r e}$ as follows:

$$
\bar{F}_{r e}=\mu N\left(1-\frac{1}{3}\left(\frac{F_{r e}}{\mu N}\right)^{2}+\frac{1}{27}\left(\frac{F_{r e}}{\mu N}\right)^{3}\right) \quad F_{r e} \leq 3 \mu N
$$

in which $\mu$ is the friction coefficient between wheel and rail, and $N$ is the normal wheel-rail contact force. For small creepages ( $F_{r e} \leq 3 \mu N$ ), the creep forces are determined by

$$
F_{c x}=\frac{F_{c x}}{F_{r e}} \bar{F}_{r e}, \quad F_{c y}=\frac{F_{c y}}{F_{r e}} \bar{F}_{r e}
$$

After the saturation at $\mathrm{B}\left(F_{r e}>3 \mu N\right)$, wheel slips on rail. The friction characteristic is negative. A typical friction force model in tribology can be used to describe the section $C D$ in Fig. 1 as follows:

$$
\left\{\begin{array}{l}
F_{c x}=a_{1}-b_{1}\left(1-e^{-c_{1} \dot{u}}\right) \\
F_{c y}=a_{2}-b_{2}\left(1-e^{-c_{2} \dot{v}}\right)
\end{array}\right.
$$

in which $a, b$ and $c$ are the constants, and $\dot{u}$ and $\dot{v}$ are the velocities of wheel slipping along $X$ and $Y$ directions.

The normal force $\left(N_{c}\right)$ of wheel-rail contact is determined using Hertz static contact theory as follows:

$$
N_{c}=C_{H} \cdot\left(\Delta D_{w r}\right)^{3 / 2}
$$

in which $\Delta D_{w r}$ is the contact deformation of the wheel and the rail, $C_{H}$ is the Hertz contact coefficient. For wheel-rail contact, it can be expressed as:

$$
C_{H}=\left[\frac{16 G_{c}^{2} r_{e}}{9\left(1-v_{c}\right)^{2}}\right]^{1 / 2}
$$

in which $G_{c}$ is the wheel-rail combined shear module, $v_{c}$ is the wheel-rail combined Poisson's ratio, and $r_{e}$ is the wheelrail calculated radius.

In the applications of Hertz static contact theory, it has been assumed that the relative displacement of a single wheelrail contact point is the deformation of the wheel-rail contact patch and is expressed as:

$$
\Delta D_{n T}=w_{R k}-w_{w k}-\mu(x)
$$

in which $w_{R k}$ is the normal displacement of rail at the contact point, $w_{w k}$ is the normal displacement of wheel at the contact point and $\mu(x)$ is the function representing the defects of the wheel and/or the rail or the track irregularities.

Finally, the normal contact force of the wheel-rail system can be expressed as:

$$
N_{c}=C_{H}\left(w_{R k}-w_{u k}-\mu(x)\right)^{3 / 2}
$$

\section{SIMULATIONS AND ANALYSIS}

Several vehicle-track systems available from Queensland Rail, Pacific National and Australian Rail and Track Co. will be simulated to examine the rail corrugation initiation and the effect of some important parameters on the rail corrugations. These parameters will be listed as follows:

- Wheelset design parameters
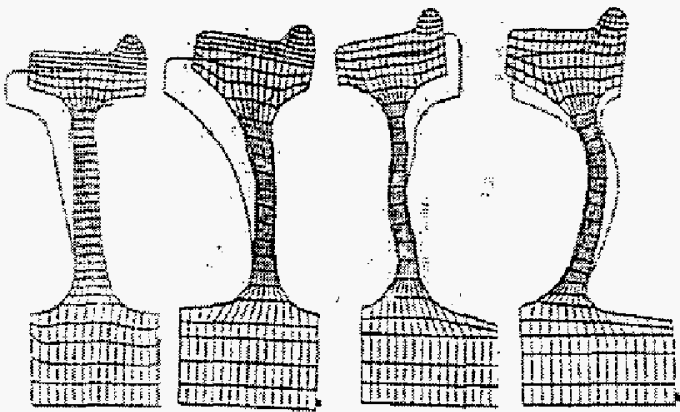

Figure 4. Different Wheel Hub Shapes 
Different wheel hub shapes shown in Figure 4 and axle dimensions would influence the natural frequencies of wheelset bending and dishing modes, causing whether or not different rail corrugations.

- Wheel and rail profiles

Different wheel and rail profiles would affect the creep forces in the wheel-rail interface, leading to the happening of rail comugations.

- Centre bowl frictions

It has been known that centre bowl friction would affect the vehicle steering on curved track. It also would affect the wheelset attack angles. The larger attack angle would cause the rail corrugation.

- Wheel-rail interface conditions

The conditions will include:

- Dry

- VHPF

- HPF

- LCF .

These conditions would influence creep forces.

- Curved track design parameters

The curved track parameters such as superelevation and curve radius, etc. can affect the creep forces in the wheelrail interface on the high or low rail, leading to the happening of rail corrugations.

\section{SOME PREVIOUS WORK RELEVENT TO RAIL CORRUGATION}

The first author had previously done some simulations relevant to rail corrugation using 3DWTSD software [14]. These results are also presented.

4.1 Rail Corrugation Caused by System Resonances

Fig. 5 shows the wheel-rail vertical dynamic factors (dynamic force divided by static wheel load) of two wheels in a wheelset. After passing through a type of cross-level irregularity, the vehicle-track system has a vertical resonance, which would lead to a long wavelength rail corrugation.

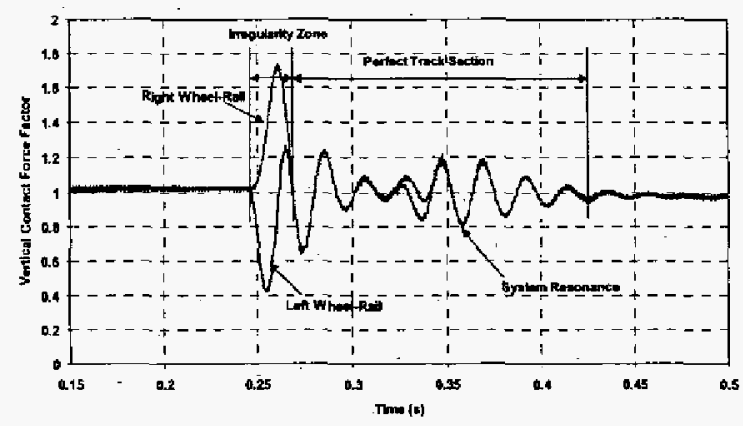

Figure 5. Rail Corrugation Due to System Resonance

\subsection{Dynamic Response Due to Rail Corrugation}

Figure 6. (a) and (b) show the rail vertical displacement at location of middle rail corrugation section and wheel-rail vertical dynamic factor. It can be seen that the rail cornugation will generate high frequency dynamic responses.

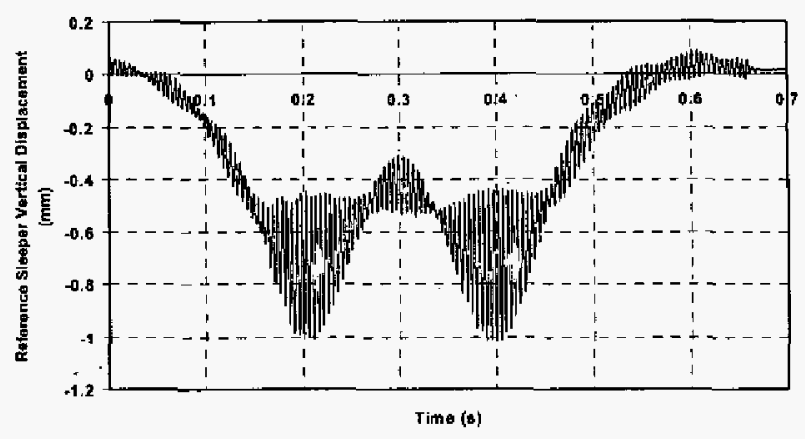

(a) Rail Vertical Displacement

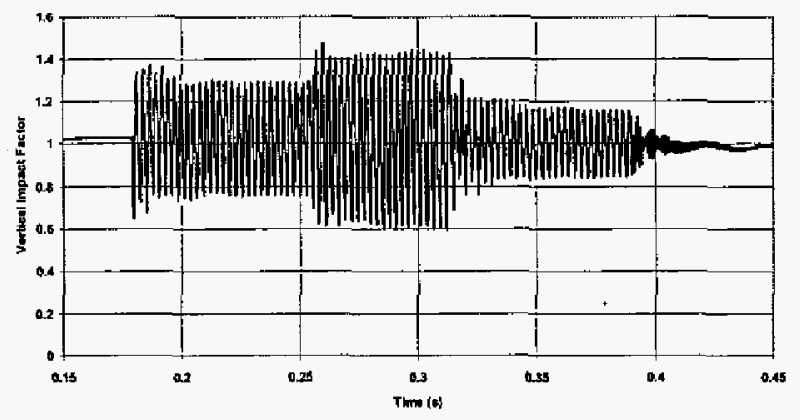

(b) Wheel-Rail Vertical Force

Figure 6. Dynamic Response Due to Rail Corrugation

\section{CONCLUSIONS}

The vehicle-track modelling for rail corrugation initiation and wheel squealing has been developed. The some parameters which would influence rail corrugation have been put forward for investigations. The further simulations are being carried out.

\section{ACKNOWLEDGMENTS}

We would like to thank the Corporative Research Centre for Railway Engineering and Technologies (Rail CRC), Australia for the funding support they have provided to investigate rail corrugation initiation.

\section{REFERENCES}

[1]. Grassie, S. L. and Kalousek, J., 1993. Rail corrugation: characteristics, causes and treatments. Proc Instn Mech Engrs, Vol. 207, 57-68.

[2]. Valdivia, A. R., 1987. A linear dynamic wear model to explain the initiating mechanism of corrugation. Proc. of $10^{\text {th }}$ IAVSD-Symposium, 493-496. 
[3]. Hempelmann, $K$, and Grob-Thebing, 20xx. A. Assessment of corrugation growth and derivation of maintenance measures by simulation using SFE AKUSRAIL. (Collected from internet)

[4]. Knothe, K. and Ripke, B., 1989. The effects of the parameters of wheelset, track and running conditions on the growth of rail corrugation. Proc. of $11^{\text {th }}$ IAVSD-Symposium, 345-356.

[5]. Tassilly, E, and Vincent, N., 1991. Rail corrugation: analytical model and field tests. Wear, Vol. 144, 163-178.

[6]. Kalousek, J. and Johnson, K. L., 1992. An investigation of short pitch wheel and rail corrugation on the Vancouver mass transit system. Proc Instn Mech Engrs, Vol. $206,127-135$.

[7]. Grassie, S. L., 1996. Short wavelength rail corrugation: field trials and measuring technology. Wear, Vol. 191, 149-160.

[8]. Clark, R. A. and Foster, P., 1983. On the mechanics of rail corrugation formation. Proc. of $8^{\text {th }}$ IAVSD-Symposium, 7285.
[9]. Ahlbeck, D. R. and Daniels, L. E., 1991. Investigation of rail corrugations on Baltimore Metro. Wear, Vol. 144, 197 210 .

[10]. Suda, Y. and Iguchi, M., 1989. Basic study of corrugation mechanism on rolling contact in order to control rail surfaces. Proc. of $11^{\text {th }}$ IAVSD-Symposium, 566-577.

[11]. Hays, W. F. and Tucker, H. G., 1991. Wheelset-track resonance as a possible source of corrugation wear. Wear, Vol. 144, $211-226$.

[12]. Matsumoto, A., Sato Y., Ono, H., Tanimoto, M., Oka, Y. and Miyauchi, E., 2002. Formation mechanism and countermeasures of rail corrugation on curved track. Wear, Vol. $253,178-184$.

[13]. Wu, T. X. and Thompson, D. J., 2002. An investigation into rail corrugation due to micro-slip under multiple wheel/rail interactions. ISVR Technical Memorandum No. 887.

[14]. Sun, Y. Q. and Dhanasekar, M., 2003. A threedimensional model for the lateral and vertical dynamics of wagon-track systems. Proc Instn Mech Engrs, Vol. 21 7, 31-45. 